\title{
Lanostane Triterpenoids from Fruiting Bodies of Ganoderma leucocontextum
}

\author{
Zhen-Zhu Zhao $\cdot$ He-Ping Chen $\cdot$ Ying Huang • \\ Zheng-Hui Li • Ling Zhang • Tao Feng • \\ Ji-Kai Liu
}

Received: 28 December 2015/ Accepted: 28 January 2016/Published online: 12 February 2016

(C) The Author(s) 2016. This article is published with open access at Springerlink.com

\begin{abstract}
Six new lanostane-type triterpenoids, namely leucocontextins S-X (1-6), together with twelve known compounds, were isolated from the fruiting bodies of Ganoderma leucocontextum. Their structures were established by MS and NMR data. Graphical Abstract
\end{abstract}
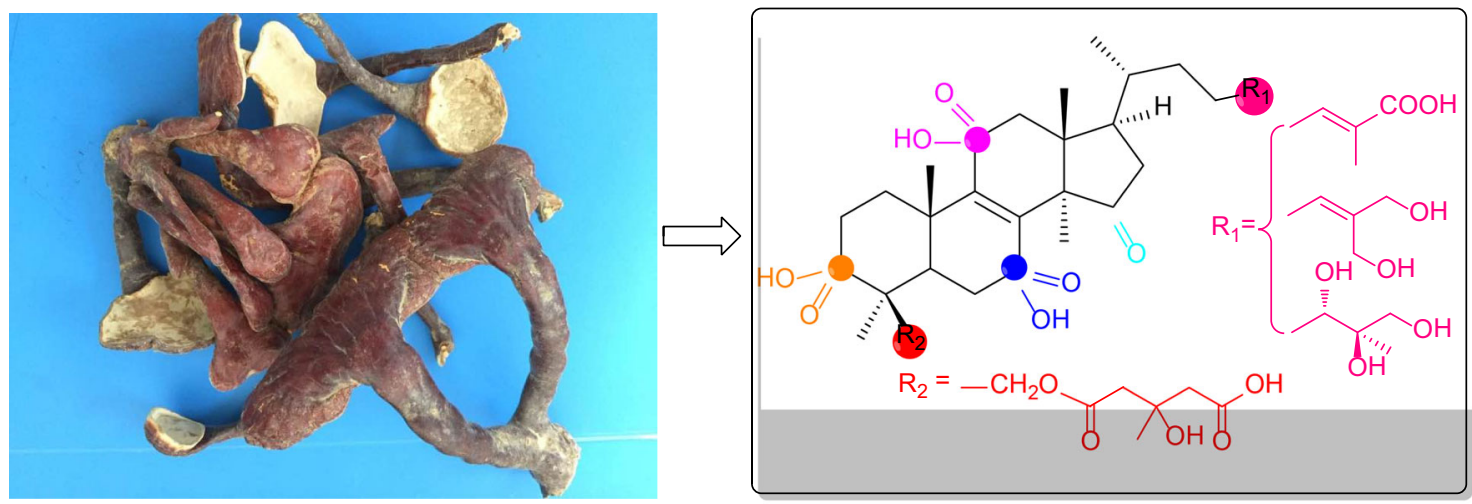

Keywords Ganoderma leucocontextum · Triterpenoids

Electronic supplementary material The online version of this article (doi:10.1007/s13659-016-0089-3) contains supplementary material, which is available to authorized users.

Z.-Z. Zhao · H.-P. Chen · Y. Huang · L. Zhang · J.-K. Liu ( $\)$ State Key Laboratory of Phytochemistry and Plant Resources in West China, Kunming Institute of Botany, Chinese Academy of Sciences, Kunming 650201, China e-mail: jkliu@mail.kib.ac.cn

Z.-Z. Zhao · H.-P. Chen · Y. Huang University of Chinese Academy of Sciences, Beijing 100049, China
Z.-H. Li · T. Feng $(\bowtie) \cdot$ J.-K. Liu School of Pharmaceutical Sciences, South-Central University for Nationalities, Wuhan 430074, China e-mail: tfeng@mail.scuec.edu.cn 


\section{Introduction}

The higher fungi of Ganoderma are documented by many ancient Chinese Medicine books as tonic drugs for their health function. Modern pharmacological researches have illuminated that the effective components of the genus Ganoderma are mainly polysaccharides [1-3] and triterpenoids [4, 5]. Triterpenoids derived from Ganoderma spp., especially lanostane-type, have been one of the hotspots for a long time and the number of triterpenoids isolated from this genus has exceeded 300 [6-10]. G. leucocontextum is a special fungus because it is mainly distributed in plateau areas and has rarely been reported for its chemical compositions [6]. In the previous research, we have reported eighteen lanostane triterpenoids from this fungus [10]. In order to further make clear the secondary constituents of $G$. leucocontextum and comprehend its intrinsic differences from other common species, a further chemical investigation on the fruiting bodies of G. leucocontextum was carried out, which led to the discovery of six new lanostane triterpenoids, along with twelve known ones (Fig. 1). This study has shed light on the similarity of chemical structures between G. leucocontextum and other species, which are mostly lanostane type triterpenoids [11]. All the new compounds were evaluated for their cytotoxicities against human myelogenous leukemia (K562), hepatocellular carcinoma (SMMC-7721) and breast cancer (MCF-7) cells lines.

\section{Results and Discussion}

Leucocontextin S (1) was isolated as colorless needles. Its molecular formula was $\mathrm{C}_{30} \mathrm{H}_{48} \mathrm{O}_{6}$ established by HREIMS $\left(\mathrm{m} / \mathrm{z}, 504.3442[\mathrm{M}]^{+}\right.$, calcd for 504.3451). The IR absorption bands at $3440,3433,1705$ and $1640 \mathrm{~cm}^{-1}$ revealed the presence of hydroxy and a conjugated carbonyl group. Analyses of the ${ }^{1} \mathrm{H}$ and ${ }^{13} \mathrm{C}$ NMR data indicated the existence of seven methyls, nine methylenes (one oxygenated), a tetrasubstituted double bond, five methines (two oxygenbonded), five $s p^{3}$ quaternary carbons (one oxygenated), and two carbonyls (Tables 1,2). The above-mentioned data suggested that compound $\mathbf{1}$ was quite similar to ganoderiol D (9) $[12,13]$, except for an extra hydroxy substituted at $\mathrm{C}-11$ of $\mathbf{1}$. This difference was confirmed by HMBC correlation of $\mathrm{H}-11\left(\delta_{\mathrm{H}} 4.52\right) / \mathrm{C}-9\left(\delta_{\mathrm{C}} 159.1\right)$ and ${ }^{1} \mathrm{H}-{ }^{1} \mathrm{H}$ COSY correlation of $\mathrm{H}-11 / \mathrm{H}-12\left(\delta_{\mathrm{H}} 1.87,2.52\right)$ (Fig. 2). The orientation of $11-\mathrm{OH}$ was established as $\alpha$ based on the correlations between $\mathrm{H}-11$ and $\mathrm{Me}-18 / \mathrm{Me}-19$ in the ROESY spectrum (Fig. 2). Furthermore, the absolute configurations of C-24 and C-25 was inferred as $S$ and $R$ via comparing with the chemical shifts of ganodermanontriol which had been synthesized in 2011 [14]. In the
${ }^{13} \mathrm{C}$ NMR data (Table 2), signals at 79.3 (C-24), 74.2 (C$25)$ and 67.5 (C-26) were nearly identical with those of ganodermanontriol [79.3 (C-24), 74.0 (C-25), 67.6 (C-26)]. Thus, structure $\mathbf{1}$ was established as $(24 S, 25 R)$ $11 \alpha, 24,25,26$-tetrahydroxy- $\alpha \alpha$-lanost-8-ene-3,7-dione.

HREIMS data gave leucocontextin $\mathrm{T}$ (2) the same molecular formula as $\mathbf{1}\left(\mathrm{C}_{30} \mathrm{H}_{48} \mathrm{O}_{6}\right)$. Analysis of the $1 \mathrm{D}$ NMR data (Tables 1,2) and HSQC displayed that $\mathbf{2}$ was an analogue of 11-oxo ganoderiol D (15) [7], while the carbonyl group at C-7 of $\mathbf{1 5}$ was reduced to a hydroxy in $\mathbf{2}$ (Supporting Information, Figure S12). And the orientation of 7-OH was $\beta$ according to the ROESY correlation of H-7 $\left(\delta_{\mathrm{H}} 4.46\right) / \mathrm{H}-5\left(\delta_{\mathrm{H}} 2.10\right)$ (Electronic supplementary material, Figure S13). Other chiral centers were same with those of $\mathbf{1}$ by comparison of the NMR data. Therefore, the structure of 2 was decided to be $(24 S, 25 R)-7 \beta, 24,25,26$ tetrahydroxy- $5 \alpha$-lanost- 8 -ene-3,11-dione.

Leucocontextin U (3) was isolated as white powder. The HRESIMS of $\mathbf{3}$ showed an ion peak at $\mathrm{m} / \mathrm{z} 507.3681$ $[\mathrm{M}+\mathrm{H}]^{+}$(calcd for $\mathrm{C}_{30} \mathrm{H}_{51} \mathrm{O}_{6}$ 507.3680), suggesting a molecular formula $\mathrm{C}_{30} \mathrm{H}_{50} \mathrm{O}_{6}$. The NMR data of $\mathbf{3}$ suggested that it was a lanostane-type triterpenoid, which displayed similar characteristic signals to $\mathbf{1}$. While the mainly difference between them was that the carbonyl located at C-3 in 1 was changed into a hydroxy group in $\mathbf{3}$. This change was supported by HMBC correlations of Me-28 $\left(\delta_{\mathrm{H}} 0.99\right) /$ $\mathrm{C}-3\left(\delta_{\mathrm{C}} 78.7\right), \mathrm{Me}-29\left(\delta_{\mathrm{H}} 0.89\right) / \mathrm{C}-3\left(\delta_{\mathrm{C}} 78.7\right)$ and ${ }^{1} \mathrm{H}-{ }^{1} \mathrm{H}$ COSY correlations of $\mathrm{H}-2\left(\delta_{\mathrm{H}} 1.72,1.69\right) / \mathrm{H}-3\left(\delta_{\mathrm{H}} 3.20\right)$ (Electronic supplementary material, Figures S18, S19). Obvious cross peaks in the ROESY spectrum between $\mathrm{H}-3$ / $\mathrm{H}-5\left(\delta_{\mathrm{H}} 1.78\right), \mathrm{H}-11\left(\delta_{\mathrm{H}} 4.50\right) / \mathrm{Me}-18\left(\delta_{\mathrm{H}} 0.71\right)$, and $\mathrm{H}-11 /$ Me-19 $\left(\delta_{\mathrm{H}} 1.25\right)$ suggested that the orientations of C-3 and $\mathrm{C}-11$ were $\beta$ and $\alpha$, respectively. The stereochemistry of other chiral centers of $\mathbf{3}$ was same with those of $\mathbf{1}$. Hence, structure of $\mathbf{3}$ was elucidated to be $(24 S, 25 R)$ $3 \beta, 11 \alpha, 24,25,26$-pentahydroxy-5 $\alpha$-lanost-8-en-7-one.

Leucocontextin V (4) had the molecular formula of $\mathrm{C}_{30} \mathrm{H}_{44} \mathrm{O}_{6}$ as determined by HRESIMS based on the ion peak at $\mathrm{m} / z 501.3207[\mathrm{M}+\mathrm{H}]^{+}$(calcd for 501.3211), requiring nine degrees of unsaturation. The 1D NMR data of $\mathbf{4}$ showed similarities to those of $\mathbf{1}$. In compound $\mathbf{4}$, the HMBC correlations from $\mathrm{H}-2\left(\delta_{\mathrm{H}} 5.28\right.$, s) to $\mathrm{C}-1\left(\delta_{\mathrm{C}}\right.$ 184.6), C-4 $\left(\delta_{\mathrm{C}} 44.2\right)$ and $\mathrm{C}-10\left(\delta_{\mathrm{C}} 42.8\right)$ suggested that an $\alpha, \beta$-unsaturated ketone group was located at ring $\mathrm{A}$ and $\mathrm{C}-1$ was oxygenated (Electronic supplementary material, Figure S26). Besides, the chemical shift of C-11 $\left(\delta_{\mathrm{C}} 81.6\right)$ of 4 was downfield shifted comparing to that of $1\left(\delta_{\mathrm{C}} 65.9\right)$. Therefore, an ether linkage was assigned between $\mathrm{C}-1$ and $\mathrm{C}-11$ based on aforementioned evidences and the degrees of unsaturation $[15,16]$. ROESY experiment showed correlations between $\mathrm{H}-11\left(\delta_{\mathrm{H}} 5.36\right) / \mathrm{Me}-18\left(\delta_{\mathrm{H}} 0.92\right)$ and $\mathrm{H}-11 / \mathrm{Me}-19\left(\delta_{\mathrm{H}} 1.43\right)$, which revealed that the orientation of $\mathrm{C}-11$ was $\alpha$. The relative configuration of 4 was same 


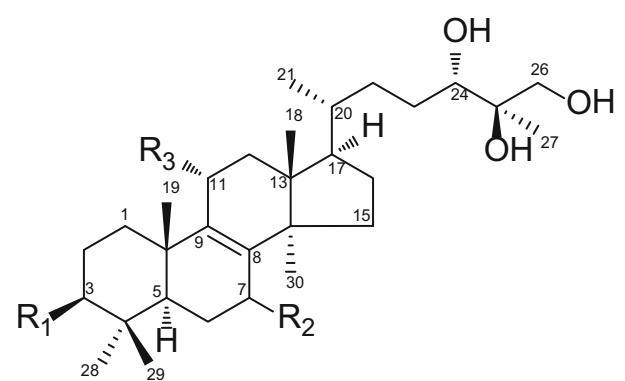

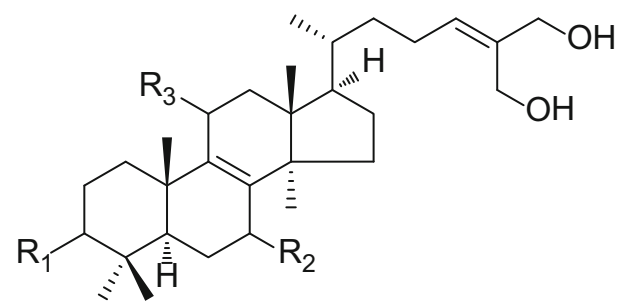

$$
\begin{array}{llll} 
& \mathrm{R}_{1} & \mathrm{R}_{2} & \mathrm{R}_{3} \\
\mathbf{1} & =\mathrm{O} & =\mathrm{O} & -\mathrm{OH} \\
\mathbf{2} & =\mathrm{O} & \beta-\mathrm{OH} & =\mathrm{O} \\
\mathbf{3} & -\mathrm{OH} & =\mathrm{O} & -\mathrm{OH} \\
\mathbf{9} & =\mathrm{O} & =\mathrm{O} & -\mathrm{H} \\
15 & =\mathrm{O} & =\mathrm{O} & =\mathrm{O} \\
16 & -\mathrm{OH} & =\mathrm{O} & -\mathrm{H}
\end{array}
$$

$$
\begin{array}{llll} 
& \mathrm{R}_{1} & \mathrm{R}_{2} & \mathrm{R}_{3} \\
\mathbf{5} & \beta-\mathrm{OH} & =\mathrm{O} & \alpha-\mathrm{OH} \\
\mathbf{7} & =\mathrm{O} & =\mathrm{O} & =\mathrm{O} \\
\mathbf{8} & =\mathrm{O} & \beta-\mathrm{OH} & =\mathrm{O} \\
10 & \beta-\mathrm{OH} & =\mathrm{O} & -\mathrm{H} \\
11 & =\mathrm{O} & =\mathrm{O} & -\mathrm{H}
\end{array}
$$

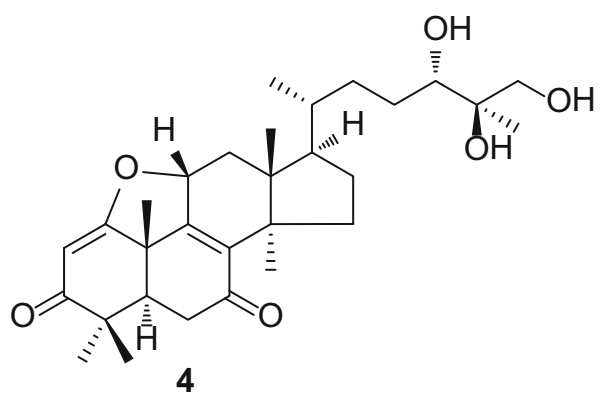

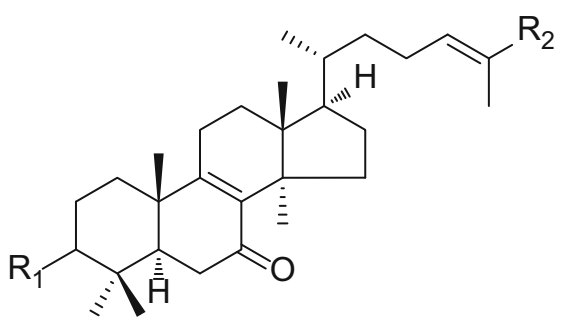

$$
\begin{array}{lll} 
& \mathrm{R}_{1} & \multicolumn{1}{c}{\mathrm{R}_{2}} \\
12 & \beta-\mathrm{OH} & -\mathrm{CHO} \\
13 & =\mathrm{O} & -\mathrm{CHO} \\
14 & \beta-\mathrm{OH} & -\mathrm{CH}_{2} \mathrm{OH}
\end{array}
$$

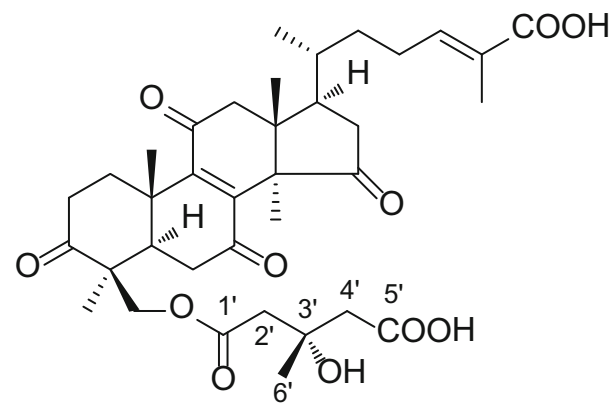

6

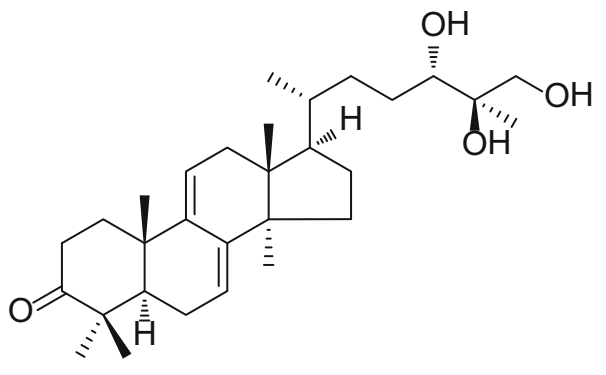

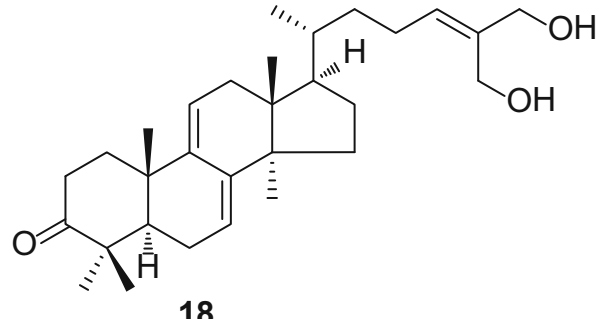

Fig. 1 Structures of compounds 1-18 
Table $1{ }^{1} \mathrm{H}$ NMR spectral data of compounds $\mathbf{1 - 5}[\delta$ in ppm, $J$ in $\mathrm{Hz}]$

\begin{tabular}{|c|c|c|c|c|c|}
\hline No. & $\mathbf{1}^{b}$ & $\mathbf{2}^{b}$ & $\mathbf{3}^{a}$ & $4^{b}$ & $\mathbf{5}^{a}$ \\
\hline \multirow[t]{2}{*}{1} & $2.30, \mathrm{~m}$ & $2.97, \mathrm{~m}$ & $2.03, \mathrm{~m}$ & & $2.02, \mathrm{~m}$ \\
\hline & $2.19, \mathrm{~m}$ & $1.72, \mathrm{~m}$ & $1.90, \mathrm{~m}$ & & $1.89, \mathrm{~m}$ \\
\hline \multirow[t]{2}{*}{2} & $2.72, \mathrm{~m}$ & $2.62, \mathrm{~m}$ & $1.72, \mathrm{~m}$ & $5.28, \mathrm{~s}$ & $1.72, \mathrm{~m}, 2 \mathrm{H}$ \\
\hline & $2.47, \mathrm{~m}$ & $2.44, \mathrm{~m}$ & $1.69, \mathrm{~m}$ & & \\
\hline 3 & & & $3.20, \mathrm{~m}$ & & $3.24, \mathrm{t}(7.6)$ \\
\hline 5 & $2.26, \mathrm{dd}(14.5,3.2)$ & $2.10, \mathrm{dd}(10.8,4.9)$ & $1.78, \mathrm{dd}(14.7,3.2)$ & $2.12, \mathrm{dd}(14.3,3.2)$ & $1.78, \mathrm{dd}(14.5,3.3)$ \\
\hline \multirow[t]{2}{*}{6} & $2.60, \mathrm{dd}(15.8,14.5)$ & $1.90, \mathrm{~m}$ & 2.59, dd $(14.7,14.7)$ & $2.53, \mathrm{dd}(17.0,14.3)$ & $2.59, \mathrm{dd}(15.7,14.5)$ \\
\hline & $2.40, \mathrm{dd}(15.8,3.2)$ & $1.69, \mathrm{~m}$ & $2.40, \mathrm{dd}(14.7,3.2)$ & $2.46, \mathrm{dd}(17.0,3.2)$ & $2.39, \mathrm{dd}(15.7,3.3)$ \\
\hline 7 & & $4.46, \mathrm{~m}$ & & & \\
\hline 11 & $4.52, \mathrm{dd}(9.1,5.2)$ & & $4.50, \mathrm{dd}(9.2,5.1)$ & $5.36, \mathrm{dd}(9.8,7.7)$ & 4.49, dd $(9.3,5.3)$ \\
\hline \multirow[t]{2}{*}{12} & $2.52, \mathrm{dd}(14.0,9.1)$ & $2.68, \mathrm{~d}(17.5)$ & $2.43, \mathrm{dd}(14.0,9.2)$ & $2.56, \mathrm{dd}(12.1,7.7)$ & $2.43, \mathrm{dd}(13.8,9.3)$ \\
\hline & 1.87, dd $(14.0,5.2)$ & $2.49, \mathrm{~d}(17.5)$ & $1.89, \mathrm{dd}(14.0,3.2)$ & $1.86, \mathrm{dd}(12.1,9.8)$ & $1.89, \mathrm{dd}(13.8,5.3)$ \\
\hline \multirow[t]{2}{*}{15} & $2.09, \mathrm{~m}$ & $1.69, \mathrm{~m}$ & $2.01, \mathrm{~m}$ & $2.00, \mathrm{~m}$ & $2.01, \mathrm{~m}$ \\
\hline & $1.61, \mathrm{~m}$ & $1.20, \mathrm{~m}$ & $1.61, \mathrm{~m}$ & $1.74, \mathrm{~m}$ & $1.60, \mathrm{~m}$ \\
\hline \multirow[t]{2}{*}{16} & $2.00, \mathrm{~m}$ & $2.10, \mathrm{~m}$ & $2.01, \mathrm{~m}$ & $2.07, \mathrm{~m}$ & $1.98, \mathrm{~m}$ \\
\hline & $1.34, \mathrm{~m}$ & $1.44, \mathrm{~m}$ & $1.34, \mathrm{~m}$ & $1.48, \mathrm{~m}$ & $1.30, \mathrm{~m}$ \\
\hline 17 & $1.57, \mathrm{~m}$ & $1.75, \mathrm{~m}$ & $1.61, \mathrm{~m}$ & $1.61, \mathrm{~m}$ & $1.58, \mathrm{~m}$ \\
\hline 18 & $0.68, \mathrm{~s}$ & $0.80, \mathrm{~s}$ & $0.71, \mathrm{~s}$ & $0.92, \mathrm{~s}$ & $0.70, \mathrm{~s}$ \\
\hline 19 & $1.39, \mathrm{~s}$ & $1.02, \mathrm{~s}$ & $1.25, \mathrm{~s}$ & $1.43, \mathrm{~s}$ & $1.25, \mathrm{~s}$ \\
\hline 20 & $1.38, \mathrm{~m}$ & $1.38, \mathrm{~m}$ & $1.41, \mathrm{~m}$ & $1.51, \mathrm{~m}$ & $1.42, \mathrm{~m}$ \\
\hline 21 & $0.93, \mathrm{~d}(6.2)$ & $0.93, \mathrm{~d}(6.4)$ & $0.97, \mathrm{~d}(6.4)$ & $1.03, \mathrm{~d}(6.3)$ & $0.98, \mathrm{~d}(6.4)$ \\
\hline \multirow[t]{2}{*}{22} & $1.83, \mathrm{~m}$ & $1.84, \mathrm{~m}$ & $1.83, \mathrm{~m}$ & $1.86, \mathrm{~m}$ & $1.54, \mathrm{~m}$ \\
\hline & $1.01, \mathrm{~m}$ & $1.01, \mathrm{~m}$ & $1.02, \mathrm{~m}$ & $1.06, \mathrm{~m}$ & $1.14, \mathrm{~m}$ \\
\hline \multirow[t]{2}{*}{23} & $1.65, \mathrm{~m}$ & $1.65, \mathrm{~m}$ & $1.83, \mathrm{~m}$ & $1.69, \mathrm{~m}$ & $2.22, \mathrm{~m}$ \\
\hline & $1.22, \mathrm{~m}$ & $1.22, \mathrm{~m}$ & $1.16, \mathrm{~m}$ & $1.25, \mathrm{~m}$ & $2.05, \mathrm{~m}$ \\
\hline 24 & $3.44, \mathrm{~d}(10.3)$ & $3.44, \mathrm{~d}(10.3)$ & $3.38, \mathrm{~d}(10.3)$ & $3.45, \mathrm{~d}(10.2)$ & $5.56, \mathrm{t}(7.3)$ \\
\hline \multirow[t]{2}{*}{26} & $3.83, \mathrm{~d}(11.1)$ & $3.83, \mathrm{~d}(11.1)$ & $4.56, \mathrm{~d}(11.1)$ & $3.84, \mathrm{~d}(11.1)$ & $4.09, \mathrm{~s}, 2 \mathrm{H}$ \\
\hline & $3.47, \mathrm{~d}(11.1)$ & $3.49, \mathrm{~d}(11.1)$ & $3.45, \mathrm{~d}(11.1)$ & $3.49, \mathrm{~d}(11.1)$ & \\
\hline 27 & $1.11, \mathrm{~s}$ & $1.10, \mathrm{~s}$ & $1.08, \mathrm{~s}$ & $1.11, \mathrm{~s}$ & $4.16, \mathrm{~s}, 2 \mathrm{H}$ \\
\hline 28 & $1.11, \mathrm{~s}$ & $1.14, \mathrm{~s}$ & $0.99, \mathrm{~s}$ & $1.20, \mathrm{~s}$ & $0.99, \mathrm{~s}$ \\
\hline 29 & $1.10, \mathrm{~s}$ & $1.07, \mathrm{~s}$ & $0.89, \mathrm{~s}$ & $1.12, \mathrm{~s}$ & $0.89, \mathrm{~s}$ \\
\hline 30 & $1.14, \mathrm{~s}$ & $1.26, \mathrm{~s}$ & $1.14, \mathrm{~s}$ & $0.96, \mathrm{~s}$ & $1.13, \mathrm{~s}$ \\
\hline
\end{tabular}

$a$ Data were measured at $600 \mathrm{MHz}$ in $\mathrm{CD}_{3} \mathrm{OD}$

$b$ Data were measured at $600 \mathrm{MHz}$ in $\mathrm{CDCl}_{3}$

with 1 (Supporting Information, Figure S27). Consequently, the structure of $\mathbf{4}$ was determined to be $(24 S, 25 R)$ $1,11 \alpha$-epoxy-24,25,26-trihydroxy-5 $\alpha$-lanosta-1,8-diene3,7-dione.

Leucocontextin $\mathrm{W}(\mathbf{5})$ possessed the molecular formula of $\mathrm{C}_{30} \mathrm{H}_{48} \mathrm{O}_{5}$ according to the HRESIMS analysis at $\mathrm{m} / \mathrm{z}$ 489.3575 $[\mathrm{M}+\mathrm{H}]^{+}$(calcd for $\mathrm{C}_{30} \mathrm{H}_{49} \mathrm{O}_{5}, 489.3575$ ). Analysis of its 1D NMR spectra showed that the structure of compound 5 was similar to ganoleucoin G (7) [6] (Tables 1,2), with the differences being that the carbonyl groups at C-3 and C-11 were reduced to hydroxy groups in 5. These changes were supported by the HMBC correlations from Me-28 $\left(\delta_{\mathrm{H}} 0.99\right)$ and Me-29 $\left(\delta_{\mathrm{H}} 0.89\right)$ to $\mathrm{C}-3\left(\delta_{\mathrm{C}}\right.$
78.9) and correlations from $\mathrm{H}-12\left(\delta_{\mathrm{H}} 2.43 ; 1.89\right)$ to $\mathrm{C}-11$ $\left(\delta_{\mathrm{C}} 65.8\right)$ (Electronic supplementary material, Figures S32, S33), as well as the HRESIMS report, which showed the molecular weight of 5 was 4 amu more than that of 7. The orientations of 3-OH and 11-OH were established as $\beta$ and $\alpha$, respectively, based on ROESY correlations of $\mathrm{H}-3\left(\delta_{\mathrm{H}}\right.$ $3.24) / \mathrm{Me}-28\left(\delta_{\mathrm{H}} 0.99\right)$ and $\mathrm{H}-11\left(\delta_{\mathrm{H}} 4.49\right) / \mathrm{Me}-19\left(\delta_{\mathrm{H}} 1.25\right)$ (Supporting Information, Figure S34). Thus the structure was defined as $3 \beta, 11 \alpha, 26,27$-tetrahydroxy-5 $\alpha$-lanosta-8,24dien-7-one.

Leucocontextin X (6) gave a molecular ion peak at $\mathrm{m} / \mathrm{z}$ $656.3188[\mathrm{M}]^{+}$in its HREIMS spectrum, indicating a molecular formula of $\mathrm{C}_{36} \mathrm{H}_{48} \mathrm{O}_{11} \quad(13$ degrees of 
Table $2{ }^{13} \mathrm{C}$ NMR spectral data of compounds $\mathbf{1}-\mathbf{5}[\delta$ in ppm, $J$ in $\mathrm{Hz}]$

\begin{tabular}{|c|c|c|c|c|c|}
\hline No. & $\mathbf{1}^{b}$ & $2^{b}$ & $3^{a}$ & $4^{b}$ & $5^{a}$ \\
\hline 1 & $34.9, \mathrm{t}$ & $34.9, \mathrm{t}$ & $35.4, \mathrm{t}$ & $184.6, \mathrm{~s}$ & $35.5, \mathrm{t}$ \\
\hline 2 & $34.8, \mathrm{t}$ & $34.2, \mathrm{t}$ & $28.2, \mathrm{t}$ & $98.1, \mathrm{~d}$ & $28.3, \mathrm{t}$ \\
\hline 3 & $214.7, \mathrm{~s}$ & $218.4, \mathrm{~s}$ & 78.7, d & $204.5, \mathrm{~s}$ & $78.9, \mathrm{~d}$ \\
\hline 4 & 47.6, s & $46.5, \mathrm{~s}$ & $40.2, \mathrm{~s}$ & $44.2, \mathrm{~s}$ & $40.3, \mathrm{~s}$ \\
\hline 5 & $50.9, \mathrm{~d}$ & $45.0, \mathrm{~d}$ & $51.9, \mathrm{~d}$ & $49.6, d$ & $52.0, \mathrm{~d}$ \\
\hline 6 & $37.7, \mathrm{t}$ & $30.1, \mathrm{t}$ & $38.0, \mathrm{t}$ & $36.4, \mathrm{t}$ & $38.1, \mathrm{t}$ \\
\hline 7 & 199.7, s & $67.3, \mathrm{~d}$ & 203.3, s & 199.1, s & 203.5, s \\
\hline 8 & $142.1, \mathrm{~s}$ & $160.3, \mathrm{~s}$ & $142.6, \mathrm{~s}$ & $137.5, \mathrm{~s}$ & $142.7, \mathrm{~s}$ \\
\hline 9 & $159.1, \mathrm{~s}$ & $140.0, \mathrm{~s}$ & $163.6, \mathrm{~s}$ & $154.7, \mathrm{~s}$ & $163.8, \mathrm{~s}$ \\
\hline 10 & $40.2, \mathrm{~s}$ & $37.8, \mathrm{~s}$ & 41.9, s & $42.8, \mathrm{~s}$ & $42.1, \mathrm{~s}$ \\
\hline 11 & $65.9, \mathrm{~d}$ & $200.6, \mathrm{~s}$ & $65.7, d$ & $81.6, d$ & $65.8, \mathrm{~d}$ \\
\hline 12 & $44.5, \mathrm{t}$ & $51.6, \mathrm{t}$ & $45.7, \mathrm{t}$ & $33.4, \mathrm{t}$ & $45.8, \mathrm{t}$ \\
\hline 13 & 47.6, s & $47.1, \mathrm{~s}$ & $47.9, \mathrm{~s}$ & $48.3, \mathrm{~s}$ & $45.9, \mathrm{~s}$ \\
\hline 14 & $48.2, \mathrm{~s}$ & $50.9, \mathrm{~s}$ & $49.5, \mathrm{~s}$ & $49.2, \mathrm{~s}$ & $49.8, \mathrm{~s}$ \\
\hline 15 & $32.8, \mathrm{t}$ & 29.2 & $34.0, \mathrm{t}$ & $29.6, \mathrm{t}$ & $34.2, \mathrm{t}$ \\
\hline 16 & $28.1, \mathrm{t}$ & $27.3, \mathrm{t}$ & $29.0, \mathrm{t}$ & $28.3, \mathrm{t}$ & $29.2, \mathrm{t}$ \\
\hline 17 & $50.0, \mathrm{~d}$ & $50.4, \mathrm{~d}$ & $51.3, \mathrm{~d}$ & $48.5, \mathrm{~d}$ & $51.4, \mathrm{~d}$ \\
\hline 18 & $17.1, \mathrm{q}$ & $17.1, \mathrm{q}$ & $17.5, \mathrm{q}$ & $16.9, \mathrm{q}$ & 17.6, q \\
\hline 19 & $19.4, \mathrm{q}$ & $17.8, \mathrm{q}$ & $20.3, \mathrm{q}$ & $18.4, \mathrm{q}$ & $20.5, \mathrm{q}$ \\
\hline 20 & $36.8, \mathrm{~d}$ & $36.8, \mathrm{~d}$ & $38.0, \mathrm{~d}$ & $36.6, d$ & $37.5, \mathrm{~d}$ \\
\hline 21 & $18.9, \mathrm{q}$ & $18.9, \mathrm{q}$ & $19.3, \mathrm{q}$ & $19.4, \mathrm{q}$ & $19.1, \mathrm{q}$ \\
\hline 22 & $33.5, \mathrm{t}$ & $33.5, \mathrm{t}$ & $34.6, \mathrm{t}$ & $33.7, \mathrm{t}$ & $37.5, \mathrm{t}$ \\
\hline 23 & $28.9, \mathrm{t}$ & $29.1, \mathrm{t}$ & $28.5, \mathrm{t}$ & $29.0, \mathrm{t}$ & $25.3, \mathrm{t}$ \\
\hline 24 & $79.3, \mathrm{~d}$ & $79.4, \mathrm{~d}$ & $77.0, \mathrm{~d}$ & $79.3, \mathrm{~d}$ & 131.1, d \\
\hline 25 & $74.2, \mathrm{~s}$ & $74.0, \mathrm{~s}$ & $75.5, \mathrm{~s}$ & $74.0, \mathrm{~s}$ & 139.1, s \\
\hline 26 & $67.5, \mathrm{t}$ & $67.8, \mathrm{t}$ & $68.9, \mathrm{t}$ & $67.8, \mathrm{t}$ & $65.7, \mathrm{t}$ \\
\hline 27 & $21.2, \mathrm{q}$ & $21.2, \mathrm{q}$ & $18.7, \mathrm{q}$ & $21.2, \mathrm{q}$ & $58.4, \mathrm{t}$ \\
\hline 28 & $21.7, \mathrm{q}$ & 27.6, q & $28.0, \mathrm{q}$ & $21.2, \mathrm{q}$ & $28.2, \mathrm{q}$ \\
\hline 29 & $25.1, \mathrm{q}$ & $20.6, \mathrm{q}$ & $15.9, \mathrm{q}$ & $29.0, \mathrm{q}$ & $16.0, \mathrm{q}$ \\
\hline 30 & $25.4, \mathrm{q}$ & $27.8, \mathrm{q}$ & $25.3, \mathrm{q}$ & $26.0, \mathrm{q}$ & $25.3, \mathrm{q}$ \\
\hline
\end{tabular}

$a$ Data were measured at $150 \mathrm{MHz}$ in $\mathrm{CD}_{3} \mathrm{OD}$

$b$ Data were measured at $150 \mathrm{MHz}$ in $\mathrm{CDCl}_{3}$

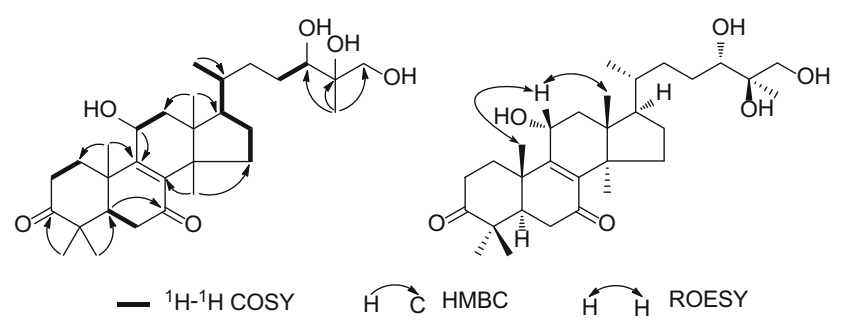

Fig. 2 Key correlations in 2D NMR spectra of compound 1

unsaturation). The 1D NMR (Table 3) displayed seven methyls $\left(\delta_{\mathrm{H}} 0.86,1.03,1.221 .25,1.36,1.43,1.63 ; \delta_{\mathrm{C}} 16.5\right.$, $18.8,22.2,12.5,27.8,18.9,21.3$ ), ten $s p^{3}$ methylenes (one
Table $3{ }^{1} \mathrm{H}$ and ${ }^{13} \mathrm{C}$ NMR spectral data of compound $6[\delta$ in ppm, $J$ in $\mathrm{Hz}$ ]

\begin{tabular}{|c|c|c|c|c|c|}
\hline No. & $\delta_{\mathrm{C}}$ & $\delta_{\mathrm{H}}$ & No. & $\delta_{\mathrm{C}}$ & $\delta_{\mathrm{H}}$ \\
\hline 1 & $35.4, \mathrm{t}$ & $\begin{array}{l}2.96, \mathrm{~m} \\
1.77, \mathrm{~m}\end{array}$ & 19 & $18.9, \mathrm{q}$ & $1.43, \mathrm{~s}$ \\
\hline 2 & $35.6, \mathrm{t}$ & $\begin{array}{l}2.66, \mathrm{~m} \\
2.61, \mathrm{~m}\end{array}$ & 20 & $36.8, \mathrm{~d}$ & $1.58, \mathrm{~m}$ \\
\hline 3 & $213.9, \mathrm{~s}$ & & 21 & $18.8, \mathrm{q}$ & $1.03, \mathrm{~d}(6.9)$ \\
\hline 4 & $51.9, \mathrm{~s}$ & & 22 & $35.4, \mathrm{t}$ & $\begin{array}{l}1.55, \mathrm{~m} \\
1.28, \mathrm{~m}\end{array}$ \\
\hline 5 & $52.6, \mathrm{~d}$ & $2.42, \mathrm{dd}(15.2,2.9)$ & 23 & $26.4, \mathrm{t}$ & $\begin{array}{l}2.31, \mathrm{~m} \\
2.19, \mathrm{~m}\end{array}$ \\
\hline 6 & $38.0, \mathrm{t}$ & $\begin{array}{l}2.87, \mathrm{~m} \\
2.61, \mathrm{~m}\end{array}$ & 24 & $143.8, \mathrm{~d}$ & $6.77, \mathrm{t}(7.0)$ \\
\hline 7 & $200.7, \mathrm{~s}$ & & 25 & $129.1, \mathrm{~s}$ & \\
\hline 8 & $147.9, \mathrm{~s}$ & & 26 & $171.7, \mathrm{~s}$ & \\
\hline 9 & $151.5, \mathrm{~s}$ & & 27 & $12.5, \mathrm{q}$ & $1.82, \mathrm{~s}$ \\
\hline 10 & $40.7, \mathrm{~s}$ & & 28 & $22.2, \mathrm{q}$ & $1.22, \mathrm{~s}$ \\
\hline 11 & $201.2, \mathrm{~s}$ & & 29 & $66.6, \mathrm{t}$ & $\begin{array}{l}4.61, \mathrm{~d}(12.2) \\
4.14, \mathrm{~d}(12.2)\end{array}$ \\
\hline 12 & $50.1, \mathrm{t}$ & $\begin{array}{l}3.08, \mathrm{~d}(15.9) \\
2.73, \mathrm{~d}(15.9)\end{array}$ & 30 & $21.3, \mathrm{q}$ & $1.63, \mathrm{~s}$ \\
\hline 13 & $45.3, \mathrm{~s}$ & & $1^{\prime}$ & $172.3, \mathrm{~s}$ & \\
\hline 14 & $58.5, \mathrm{~s}$ & & $2^{\prime}$ & $46.2, \mathrm{t}$ & $2.67, \mathrm{~s}, 2 \mathrm{H}$ \\
\hline 15 & $210.5, \mathrm{~s}$ & & $3^{\prime}$ & $70.8, \mathrm{~s}$ & \\
\hline 16 & $41.1, \mathrm{t}$ & $\begin{array}{l}2.89, \mathrm{~m} \\
1.89, \mathrm{dd}(18.3,8.1)\end{array}$ & $4^{\prime}$ & $46.2, \mathrm{t}$ & $2.61, \mathrm{~s}, 2 \mathrm{H}$ \\
\hline 17 & $46.1, d$ & $2.27, \mathrm{~m}$ & $5^{\prime}$ & $175.9, \mathrm{~s}$ & \\
\hline 18 & $16.5, \mathrm{q}$ & $0.86, \mathrm{~s}$ & $6^{\prime}$ & $27.8, \mathrm{q}$ & $1.36, \mathrm{~s}$ \\
\hline
\end{tabular}

$\delta_{\mathrm{H}}$ Data were measured at $600 \mathrm{MHz}$ in $\mathrm{CD}_{3} \mathrm{OD}$

$\delta_{\mathrm{C}}$ Data were measured at $150 \mathrm{MHz}$ in $\mathrm{CD}_{3} \mathrm{OD}$

oxygenated, $\left.\delta_{\mathrm{C}} 66.6\right)$, three $s p^{3}$ methines, five $s p^{3}$ quaternary carbons (one oxygenated, $\delta_{\mathrm{C}} 70.8$ ), a tetrasubstituted and a trisubstituted double bonds as well as seven carbonyls $\left(\delta_{\mathrm{C}} 171.7,172.3,175.9,200.7,201.2,210.5\right.$ and 213.9). Furthermore, the aforementioned data, together with $\mathrm{HMBC}$ and ${ }^{1} \mathrm{H}-{ }^{1} \mathrm{H}$ COSY spectral signals, showed that structure of 6 was closely related to ganoleucion L [6], difference being that the (4-carboxy-3-hydroxy-3methylbutanoyl)oxy group was substituted at C-29 based on HMBC correlations from $\mathrm{H}-29\left(\delta_{\mathrm{H}} 4.61\right.$ and 4.62) to $\mathrm{C}-1^{\prime}\left(\delta_{\mathrm{C}} 172.3\right)$ (Supporting Information, Figure S39). The absolute configuration of $\mathrm{C}^{-3^{\prime}}$ was inferred as $S$ by comparing the carbon chemical shifts of (4-carboxy-3-hydroxy3-methylbutanoyl)oxy group between $\mathbf{6}$ and those of ganoleucion L in the same NMR solvent (Supporting Information, Figure S43). As a result, the structure of 6 was elucidated as (24E)-29-O-((3S)-4-carboxy-3-hydroxy-3- 
methylbutanoyl)-3,7,11,15-tetraoxo-5 $\alpha$-lanosta-8,24-dien26-oic acid.

Twelve known lanostane-type triterpeniods, ganoleucoins G (7) [6] and I (8) [6], ganoderiols D (9) [12], E (10) [12], F (18) [12], H (16) [12] and J (11) [13], lucialdehyde B (13) [17], lucidadiol (14) [18], lucidal (12) [18], 11-oxo ganoderiol D (15) [7] and ganodermanontriol (17) [12, 14] were also obtained from this fungus. Their structures were identified by 1D NMR spectrum as well as comparison with reported data.

Compounds 1-6 were evaluated for inhibitory activities against K562, SMMC-7721 and MCF-7 cell lines. The cytotoxicity was determined by using MTS method. Unfortunately, none of them showed significant activity.

\section{Experimental Section}

\subsection{General Experimental Procedures}

Optical rotations were obtained on a JASCO P-1020 digital polarimeter (Horiba, Kyoto, Japan). UV spectra were recorded on a Shimadzu UV-2401PC (Shimadzu, Kyoto, Japan). 1D and 2D NMR spectra were obtained on a Bruker Avance III $600 \mathrm{MHz}$ spectrometer (Bruker Biospin $\mathrm{GmbH}$, Karlsruhe, Germany). HREIMS was measured on Waters Xevo TQ-S and Waters Autospec Premier P776 mass spectrometers (Waters, Milford, MA, USA). HRESIMS were recorded on an Agilent 6200 Q-TOF MS system (Agilent Technologies, Santa Clara, CA, USA). Melting points were measured on an X-4 microscopic melting point meter (Yuhua Instrument Co., Ltd, Gongyi, China). Sephadex LH-20 (Amersham Biosciences, Upssala, Sweden) and silica gel (Qingdao Haiyang Chemical Co., Ltd) were used for column chromatography (CC). Medium Pressure Liquid Chromatography (MPLC) was performed on a Büchi Sepacore System equipping with pump manager C-615, pump modules C-605 and fraction collector C-660 (Büchi Labortechnik AG, Flawil, Switzerland), and columns packed with Chromatorex C-18 (40-75 $\mu \mathrm{m}$, Fuji Silysia Chemical Ltd., Kasugai, Japan). Preparative High Performance Liquid Chromatography (prep-HPLC) was performed on an Agilent 1260 liquid chromatography system equipped with Zorbax SB-C18 columns (5 $\mu \mathrm{m}$, $9.4 \mathrm{~mm} \times 150 \mathrm{~mm}$ or $21.2 \mathrm{~mm} \times 150 \mathrm{~mm}$ ) and a DAD detector (Agilent Technologies, Santa Clara, CA, USA).

\subsection{Fungal Material}

The fungus G. leucocontextum were collected in Nyingchi, Tibet, China in 2014, and identified by professor Yu-Cheng Dai (Beijing Forestry University). A voucher specimen of
G. leucocontextum was deposited in the Herbarium of Kunming Institute of Botany, Chinese Academy of Sciences (No. HFC 20140613).

\subsection{Extraction and Isolation}

The air-dried and powdered fruiting bodies of G. leucocontextum $(2.5 \mathrm{~kg})$ was macerated three times with $95 \%$ methanol. The extract was evaporated under reduced pressure and partitioned between EtOAc and water four times to give a crude extract $(65 \mathrm{~g})$. The crude extract was subject to MPLC with a stepwise gradient of $\mathrm{MeOH} / \mathrm{H}_{2} \mathrm{O}$ (v/v 40:60-100:0) to afford nine fractions (A-I).

Fraction D (10.5 g) was separated by Sephadex LH-20 $(\mathrm{MeOH})$ to give two major subfractions (D1-D2). Subfraction D1 was separated on silica gel CC using a petroleum ether-acetone gradient solvent system (v/v, 4:1-2:1) to obtain three subfractions (D1a-D1c). Subfraction D1a and D1b were separated by Sephadex LH-20 (acetone) to afford three subfractions (D1a1-D1a3 and D1b1-D1b3), respectively. Each part was purified on prepHPLC (MeCN-H ${ }_{2} \mathrm{O} 20-45 \%, 25 \mathrm{~min}$ ) to yield compounds 10 (8.0 mg), 11 (9.4 mg), 12 (7.3 mg), 13 (11.5 mg), 14 $(21.0 \mathrm{mg})$, and 18 (15.4 mg). Fraction E (12.5 g) was separated on Sephadex LH-20 (MeOH) to give four subfractions (E1-E4). E1 was subjected to silica gel CC with a petroleum ether-acetone gradient solvent system (v/v, $4: 1-2: 1)$ to obtain six subfractions (E1a-E1f). Compounds $7(1.5 \mathrm{mg})$ and $8(3.8 \mathrm{mg})$ were isolated from E1e by prepHPLC (MeCN-H $\left.\mathrm{H}_{2} \mathrm{O} 24-39 \%, 30 \mathrm{~min}\right)$. E1f was subjected on prep-HPLC (MeCN- $\left.\mathrm{H}_{2} \mathrm{O} 21-41 \%, 25 \mathrm{~min}\right)$ to afford 6 (19.7 mg), 1 (5.3 mg), 2 (4.3 mg).

Fraction F (11.3 g) was separated on Sephadex LH-20 $(\mathrm{MeOH})$ to give three sections $(\mathrm{F} 1-\mathrm{F} 3)$ and $\mathrm{F} 1$ was separated by MPLC with $\mathrm{MeOH}-\mathrm{H}_{2} \mathrm{O}$ (v/v 40:60-80:20) to give four subfractions (F1a-F1d). Then fraction F1b was subjected to Sephadex LH-20 (acetone) to give three subfractions (F1b1-F1b3) and each of them was purified by prep-HPLC with the mobile phase $\mathrm{MeCN} / \mathrm{H}_{2} \mathrm{O}(25-45 \%$, $25 \mathrm{~min})$ to afford compounds $3(2.0 \mathrm{mg}), \mathbf{4}(2.9 \mathrm{mg}), \mathbf{5}$ (3.1 mg), 9 (24.5 mg), 15 (8.9 mg), 16 (13.2 mg) and 17 (11.0 mg).

\subsection{Leucocontextin S (1)}

Colorless needles; m.p. 208.2-209.4; $[\alpha]_{\mathrm{D}}^{17}-18.13$ (c 0.40, $\mathrm{MeOH})$. UV $(\mathrm{MeOH}) \lambda_{\max } \mathrm{nm}(\log \varepsilon): 250.8$ (3.82). IR $(\mathrm{KBr}) v_{\max } \mathrm{cm}^{-1}: 3440,3433,2955,2924,2853,1705$, 1640, 1461, 1383, 1112, 1036; ${ }^{1} \mathrm{H}$ NMR $(600 \mathrm{MHz}$, $\left.\mathrm{CDCl}_{3}\right)$ and ${ }^{13} \mathrm{C} \mathrm{NMR}\left(150 \mathrm{MHz}, \mathrm{CDCl}_{3}\right)$ data, Tables 1, 2, HREIMS $m / z$ : $504.3442[\mathrm{M}]^{+}$(calcd for $\mathrm{C}_{30} \mathrm{H}_{48} \mathrm{O}_{6}$, 504.3451). 
3.5 Leucocontextin T (2)

White powder; $[\alpha]_{\mathrm{D}}^{22}+141.22(c \quad 0.30, \mathrm{MeOH})$. UV $(\mathrm{MeOH}) \lambda_{\max } \mathrm{nm}(\log \varepsilon): 254.0$ (3.75). IR (KBr) $v_{\max }$ $\mathrm{cm}^{-1}: 3424,2969,2938,2884,1701,1657,1636,1463$, 1384, 1040; ${ }^{1} \mathrm{H}$ NMR (600 MHz, $\mathrm{CDCl}_{3}$ ) and ${ }^{13} \mathrm{C}$ NMR $\left(150 \mathrm{MHz}, \mathrm{CDCl}_{3}\right)$ data, Tables 1 and 2, HREIMS $\mathrm{m} / \mathrm{z}$ : $504.3459[\mathrm{M}]^{+}$(calcd for $\mathrm{C}_{30} \mathrm{H}_{48} \mathrm{O}_{6}, 504.3451$ ).

\subsection{Leucocontextin U (3)}

White powder; $[\alpha]_{\mathrm{D}}^{17}-29.44(c 0.06, \mathrm{MeOH})$. UV $(\mathrm{MeOH})$ $\lambda_{\max } \mathrm{nm}(\log \varepsilon): 252.4$ (3.56). IR (KBr) $v_{\max } \mathrm{cm}^{-1}: 3446$, 2960, 2923, 1647, 1461, 1384, 1035; ${ }^{1} \mathrm{H}$ NMR $(600 \mathrm{MHz}$, $\left.\mathrm{CD}_{3} \mathrm{OD}\right)$ and ${ }^{13} \mathrm{C}$ NMR $\left(150 \mathrm{MHz}, \mathrm{CD}_{3} \mathrm{OD}\right)$ data, Tables 1 and 2, HRESIMS $m / z$ : $507.3681[\mathrm{M}+\mathrm{H}]^{+}$(calcd for $\mathrm{C}_{30} \mathrm{H}_{51} \mathrm{O}_{6}$, 507.3680).

\subsection{Leucocontextin V (4)}

White powder; $[\alpha]_{\mathrm{D}}^{24}-73.83(c 0.20, \mathrm{MeOH})$. UV $(\mathrm{MeOH})$ $\lambda_{\max } \mathrm{nm}(\log \varepsilon): 257.0$ (4.02), 382.0 (2.89). IR (KBr) $v_{\max }$ $\mathrm{cm}^{-1}: 3440,2962,2926,1632,1461,1384,1042 ;{ }^{1} \mathrm{H}$ NMR $\left(600 \mathrm{MHz}, \mathrm{CDCl}_{3}\right)$ and ${ }^{13} \mathrm{C} \mathrm{NMR}\left(150 \mathrm{MHz}, \mathrm{CDCl}_{3}\right)$ data, Tables 1 and 2, HRESIMS m/z: $501.3207[\mathrm{M}+\mathrm{H}]^{+}($calcd for $\mathrm{C}_{30} \mathrm{H}_{45} \mathrm{O}_{6}, 501.3211$ ).

\subsection{Leucocontextin W (5)}

Colorless needles; m.p. 179.3-186.4; $[\alpha]_{\mathrm{D}}^{23}+1.75(c 0.01$, $\mathrm{MeOH})$. UV (MeOH) $\lambda_{\max } \mathrm{nm}(\log \varepsilon): 252.0$ (3.80). IR (KBr) $v_{\max } \mathrm{cm}^{-1}: 3446,2955,2924,2853,1641,1461$, 1383, 1112, 1036; ${ }^{1} \mathrm{H}$ NMR (600 MHz, $\left.\mathrm{CD}_{3} \mathrm{OD}\right)$ and ${ }^{13} \mathrm{C}$ NMR (150 MHz, $\left.\mathrm{CD}_{3} \mathrm{OD}\right)$ data, Tables 1, 2, HRESIMS $m /$ $z: 489.3575[\mathrm{M}+\mathrm{H}]^{+}\left(\right.$calcd for $\left.\mathrm{C}_{30} \mathrm{H}_{49} \mathrm{O}_{5}, 489.3575\right)$.

\subsection{Leucocontextin X (6)}

Yellow powder; $[\alpha]_{\mathrm{D}}^{21}+111.92(c \quad 0.26, \mathrm{MeOH})$. UV $(\mathrm{MeOH}) \lambda_{\max } \mathrm{nm}(\log \varepsilon): 213.6$ (4.16), 253.4 (3.80). IR (KBr) $v_{\max } \mathrm{cm}^{-1}: 3433,3086,2976,2936,1746,1701$, 1684, 1462, 1414, 1385, 1229, 1184, 1014. ${ }^{1} \mathrm{H}$ NMR $\left(600 \mathrm{MHz}, \mathrm{CD}_{3} \mathrm{OD}\right)$ and ${ }^{13} \mathrm{C}$ NMR $\left(150 \mathrm{MHz}, \mathrm{CD}_{3} \mathrm{OD}\right)$ data, Table 3, HREIMS m/z: $656.3188[\mathrm{M}]^{+}$(calcd for $\left.\mathrm{C}_{36} \mathrm{H}_{48} \mathrm{O}_{11}, 656.3197\right)$.

\subsection{Cytotoxicity Assay}

The cytotoxicity against K562, SMMC-7721 and MCF-7 cells lines of compounds 1-6 were tested by using MTS method. MTS [3-(4,5-dimethylthiazol-2-yl)-5(3-carboxymethoxyphenyl)-2-(4-sulfopheny)- $2 H$-tetrazolium]is an analogue of MTT [19], which can be reduced into soluble formazan by succinate dehydrogenase in mitochondria of living cells. Moreover, the optical density value of formazan $(490 \mathrm{~nm}$ ) is proportional to the number of living cells (Electronic supplementary material, Table S1).

Acknowledgments This work was financially supported by National Key Technology Support Program, China (2013BAI11B02), NSFC-joint fund of Yunnan Province (81561148013), and National Natural Science Foundation of China (81373289).

\section{Compliance with Ethical Standards}

Conflict of interest The authors declare no conflict of interest.

Open Access This article is distributed under the terms of the Creative Commons Attribution 4.0 International License (http:// creativecommons.org/licenses/by/4.0/), which permits unrestricted use, distribution, and reproduction in any medium, provided you give appropriate credit to the original author(s) and the source, provide a link to the Creative Commons license, and indicate if changes were made.

\section{References}

1. S. Zhang, S. Nie, D. Huang, J. Huang, Y. Feng, M. Xie, J. Agric. Food Chem. 62, 9296-9304 (2014)

2. T.G. Pillai, P. Uma Devi, Mutat. Res. Genet. Toxicol. Environ. Mutagen. 751, 109-115 (2013)

3. P. Saravanakumar, V. Karthikeyan, S. Patharajan, T. Kannan, K. Sathya, P.T. Kalaichelvan, Pharmacologyonline 1, 958-970 (2010)

4. J.L. Rios, I. Andujar, M.C. Recio, R.M. Giner, J. Nat. Prod. 75, 2016-2044 (2012)

5. S.F. Yeh, K.C. Lee, M.S. Shiao, Proc. Natl. Sci. Counc. Repub. China Part A 11, 129-134 (1987)

6. K. Wang, L. Bao, W.K. Ma, J. Xiong, W. Han, W. Wang, H. Yin, J. Liu, Nat. Prod. 78, 1977-1989 (2015)

7. S.S. Zhang, Q.Y. Ma, S.Z. Huang, H.F. Dai, Z.K. Guo, Z.F. Yu, Y.X. Zhao, Phytochemistry 110, 133-139 (2015)

8. Z.Z. Zhao, R.H. Yin, H.P. Chen, T. Feng, Z.H. Li, Z.J. Dong, B.K. Cui, J.K. Liu, J. Asian Nat. Prod. Res. 17, 750-755 (2015)

9. X. Peng, J. Liu, J. Xia, C. Wang, X. Li, Y. Deng, N. Bao, Z. Zhang, M.H. Qiu, Phytochemistry 114, 137-145 (2015)

10. Z.Z. Zhao, H.P. Chen, Z.H. Li, Z.J. Dong, X. Bai, Z.Y. Zhou, T. Feng, J.K. Liu, Fitoterapia 109, 91-98 (2016)

11. Q. Xia, H. Zhang, X. Sun, H. Zhao, L. Wu, D. Zhu, G. Yang, Y. Shao, X. Zhang, X. Mao, L. Zhang, Molecules 19, 17478-17535 (2014)

12. T. Nishitoba, K. Oda, H. Sato, S. Sakamura, Agric. Biol. Chem. 52, 367-372 (1988)

13. J.Q. Liu, C.F. Wang, Y. Li, H.R. Luo, M.H. Qiu, Planta Med. 78, 368-376 (2012)

14. E.M. Kennedy, S.J. P'Pool, J. Jiang, D. Sliva, R.E. Minto, J. Nat. Prod. 74, 2332-2337 (2011)

15. A.H. Banskota, Y. Tezuka, K.Q. Tran, K. Tanaka, I. Saiki, S. Kadota, Chem. Pharm. Bull. 48, 496-504 (2000)

16. R.M. Silverstein, F.X. Webster, D.J. Kiemle, Spectrometric identification of organic compounds, 7th edn. (Wiley, New York, 2005), p. 225

17. J.J. Gao, B.S. Min, E.M. Ahn, N. Nakamura, H.K. Lee, M. Hattori, Chem. Pharm. Bull. 50, 837-840 (2002)

18. A.G. González, F. León, A. Rivera, C.M. Muñoz, J. Bermejo, J. Nat. Prod. 62, 1700-1701 (1999)

19. T. Mosmann, J. Immunol. Methods 65, 55-63 (1983) 